\title{
Revisión del diseño de sitios web de gobierno desde una perspectiva comunicacional
}

Government websites design. A review from a communication perspective

Florencia Martín y Edith Noemí Lovos

Centro Interdisciplinario de Estudios sobre Derechos, Inclusión y Sociedad; Universidad Nacional de Río Negro (Argentina) flormartinlav@gmail.com; elovos@unrn.edu.ar

\section{Resumen}

Las TIC en los espacios de gobierno se asocian al denominado e-gobierno, un concepto que no se restringe sólo a cuestiones tecnológicas, sino que para su análisis pueden tomarse diversas perspectivas, entre otras la comunicacional. En el caso particular de los sitios web de gobierno resulta importante analizar la forma en la que se produce la interacción con los ciudadanos. En este trabajo se propone utilizar el análisis del discurso como una metodología que permita revisar el diseño de un sitio web de gobierno electrónico desde la perspectiva comunicacional. Así mismo, se presentan y discuten los resultados preliminares, sobre el análisis de un sitio web de gobierno electrónico del área de educación de una provincia de la patagonia argentina. 
Palabras claves: sitios web; gobierno electrónico; comunicación.

\section{Abstract}

ICTs in the areas of government are associated with the so-called e-government, a concept that is not restricted only to technological issues, but for its analysis can take diverse perspectives, including communication. In the particular case of government websites, it is important to analyze the way in which interaction with citizens occurs. In this paper it is proposed to use discourse analysis as a methodology to review the design of an e-government website from a communicational perspective. Likewise, preliminary results are presented and discussed, on the analysis of an e-government website of the education area of a province of Argentine Patagonia.

Keywords: web site; electronic government; communication.

Cada día se vuelve más evidente el impacto que las tecnologías de la información y la comunicación (TIC) tienen en la sociedad, posibilitando nuevas formas de relacionarnos, de comunicarnos, de acceder y producir información. En este sentido los gobiernos no escapan a esta realidad, y las TIC en este ámbito adquieren un rol relevante vinculadas al denominado egobierno o gobierno electrónico. Concepto que no se restringe al campo tecnológico, sino que para su análisis pueden tomarse diversas perspectivas: comunicacional, cultural, entre otras.

En el campo comunicacional el estudio de la interacción entre los dispositivos tecnológicos y las personas ha sido un punto de relevancia para diferentes teóricos, entre ellos Lorenzo Vilches (2001) y Carlos Scolari (2015). Este último, entiende que el concepto de interactividad puede asumir diferentes sentidos:

Hay interactividad en las comunicaciones sujeto-sujeto pero también en los intercambios entre un sujeto y un dispositivo tecnológico. En este segundo ejemplo la interactividad se desarrolla en la interfaz, que se podría definir como el lugar de la interacción (Scolari, 2004: 94).

Por su parte, Lorenzo Vilches (2001) advierte también que:

La interface no es un complemento sino es el centro de la interacción, y resulta una de las nuevas formas de comunicación virtual, es decir, no sólo es una herramienta, sino un proceso interactivo entre usuario y máquina (Vilches, 2001: 219).

A partir de estas visiones, en este trabajo se propone analizar cómo los sitios web de gobierno delinean esa interacción con los usuarios de los mismos. Desde la perspectiva tecnológica 
Rodríguez et al;(2015), señala que las TIC posibilitan dos espacios de comunicación virtual en el marco gubernamental: uno hacia adentro de la administración pública (comunicación interna) y otro hacia afuera de la administración pública (comunicación externa), que pueden subclasificarse como:

-Comunicación Interna: Gobierno a Gobierno (G2G) y Gobierno a Empleados (G2E)

-Comunicación Externa: Gobierno-Ciudadanos (G2C), Gobierno-Visitantes (G2V) y GobiernoEmpresas (G2B)

Dentro de las G2C se encuentran los sitios web de gobiernos, que son la base para el desarrollo del gobierno electrónico, posibilitando una representación en el espacio web de una institución del estado y una nueva forma de comunicación. En el caso particular de Argentina, un estudio sobre comunicación gubernamental en el país (Amadeo Belén, 2007), y en relación a los sitios web de gobierno, señalaba por una parte la importancia que los distintos gobiernos provinciales le otorgaban a este medio y por otra la necesidad de mejorar cuestiones como: diseño, contenidos y criterios estéticos, sino y principalmente la forma de interactuar con los ciudadanos, ya que solo proveían información vertical.

Atendiendo a este último punto, en este trabajo se propone el uso del análisis del discurso como una estrategia que permita revisar el diseño de un sitio web de gobierno electrónico combinando los aportes de la ciencia informática con los desarrollos del campo comunicacional. Así mismo, se presentan resultados de un análisis preliminar, usando esta estrategia, sobre un grupo de usuarios del sitio de gobierno del Ministerio de Educación y Derechos Humanos de la Provincia de Río Negro en la Patagonia Argentina.

Conceptualmente, se ha optado por un enfoque comunicacional de construcción de sentido (Ceraso, 2006), entendiendo que comunicar implica crear lazos, pensar a la comunicación como una relación con otras personas que sienten, que quieren, que piensan, que entienden que la recepción humana no es automatizada ni mecánica. Cada sujeto que interviene en el proceso de comunicación pone en práctica su propia forma de interpretar los códigos y de ver el mundo, es decir sus competencias culturales: la capacidad de conocer el mundo que lo rodea y de adaptarse a él y a sus reglas (Ceraso, 2006).

Es importante señalar que este trabajo forma parte del desarrollo de una tesis de grado de la Lic. en Comunicación Social de la Universidad Nacional de Río Negro, Argentina (UNRN), que tiene por objetivo identificar líneas de acción para el rediseño, desde un enfoque comunicacional de construcción de sentido, del sitio web del Ministerio de Educación y Derechos Humanos de la provincia de Río Negro. La misma se lleva adelante en el marco del Centro Interdisciplinario de Estudios sobre Derechos Inclusión y Sociedad (CIEDIS) de la UNRN. 


\section{Sitios web de gobierno}

Desde el campo de la ciencia informática se vienen llevado adelante diferentes investigaciones sobre sitios gubernamentales, advirtiendo que si bien éstos comparten características similares a las de cualquier otro sitio web, tienen un objetivo bien definido que es el de: "gestionar de una manera distinta la información, en compartir los datos e información para el uso público de manera más racional y controlada, en cambiar la forma en que los funcionarios y empleados públicos interactúan entre ellos y con el público en general" (Reynoso et al., 2013). Y para alcanzar esa meta, los sitios web de gobierno deben contemplar algunas características vinculadas al diseño tales como: funcionalidad, usabilidad, navegabilidad, amigabilidad, veracidad. (Giulianelli et al., 2008). Por su parte Rocío Rodríguez (2011) en una investigación sobre medición de calidad de gobierno electrónico en el ámbito municipal de la provincia de Buenos Aires (Argentina), sostiene que además de éstas, deben tomarse en cuenta otras características vinculadas específicamente al contenido del sitio web, tales como: Información, Servicios y Veracidad (parámetros aplicables al sitio web que provea servicios a través de la web), Transparencia y Participación Ciudadana.

Por otra parte, en el campo de las ciencias de la comunicación se vienen produciendo cambios que dejan atrás la idea de una comunicación verticalista, o de aguja hipodérmica como se llamó en las primeras teorías de la comunicación, para dar lugar a nuevas formas de pensar como es la de producción de sentido (Ceraso, 2006), lo cual implica comprender a la comunicación en conjunto como un quehacer colectivo, en el cual estén incluidos todos los actores sociales. Esta perspectiva se puede vincular con los desarrollos de Carlos Scolari (2015) quien estudia a los medios y las nuevas formas de comunicación digital dentro de un entorno de ecología de medios, entendiéndose en relación con otros. Como advierte Mc Luhan (1964): "ningún medio puede adquirir significado o existencia solo sino exclusivamente en interacción con otros medios". A partir de esto Scolari (2015), comienza a pensar a los medios como especies que conviven en un ecosistema y establecen relaciones entre sí y que las tecnologías de la comunicación generan ambientes que afectan a los sujetos que las utilizan. En tiempos de las denominadas tecnologías 2.0 o web social, estas plataformas de comunicación se caracterizan por la autogeneración de contenido y por forjar espacios dinámicos e interactivos en donde el usuario pueda compartir información y recursos con otros. Si pensamos a la comunicación en este sentido, resulta importante recuperar los estudios de Lorenzo Vilches (2001) quien hace hincapié en la interface permitiendo entender que:

La interactividad en el campo de los medios no puede centrarse en la comunicación de masas, y se ha de comprender otros conceptos como el de interface. Desde el momento en que un 
usuario utiliza una interface objeto/software, permite una interacción entre usuario y máquina la cual va a estar determinada por el entorno informático, un lenguaje y por ende un entorno cultural que establecerá la interacción que exista entre ellos (Vilches, 2001: 218).

Esto permite entender a la interface no como un complemento sino como el centro de la interacción, que a su vez forma parte de las nuevas formas de comunicación virtual, es decir, no es sólo una herramienta, sino un proceso interactivo entre usuario y máquina.

\section{Análisis del discurso}

El Análisis del Discurso, como explica Magariños de Morentín (1996), es una metodología cualitativa cuyo objetivo consiste en establecer el contenido semántico de los conceptos correspondientes a los términos efectivamente utilizados en determinados textos. El mismo autor explica que el origen de esta metodología proviene, por una parte del estructuralismo norteamericano, a través de los continuadores de la obra de Zellig Harris (1954) y, por otra, de la escuela francesa de análisis de discurso, originada en los trabajos lingüísticos de Michel Pêcheux $(1969,1975)$.

Fundamentalmente, explica Magariños (1996) se diferencia del análisis del contenido al no admitir conocimiento a priori de ninguna clase, en cuanto al contenido semántico del lenguaje, sino que se propone explicar, respecto de cada término, de qué modo construye tal contenido o significación en función de su uso en el contexto material y positivo en el que aparece. El significado es una construcción cuya materia prima es lo efectivamente dicho en el discurso, sin que sea lícito acudir al conocimiento que pueda tenerse de la historia de las ideas o de la cultura de determinada comunidad (salvo que se aporten los textos correspondientes).

\section{Metodología}

El sitio web analizado corresponde al Ministerio de Educación y Derechos Humanos de la Provincia de Río Negro, y al mismo puede es posible acceder a través de la url: http://www.educacion.rionegro.gov.ar/. En la figura 1 se muestra una captura de pantalla del mismo. 


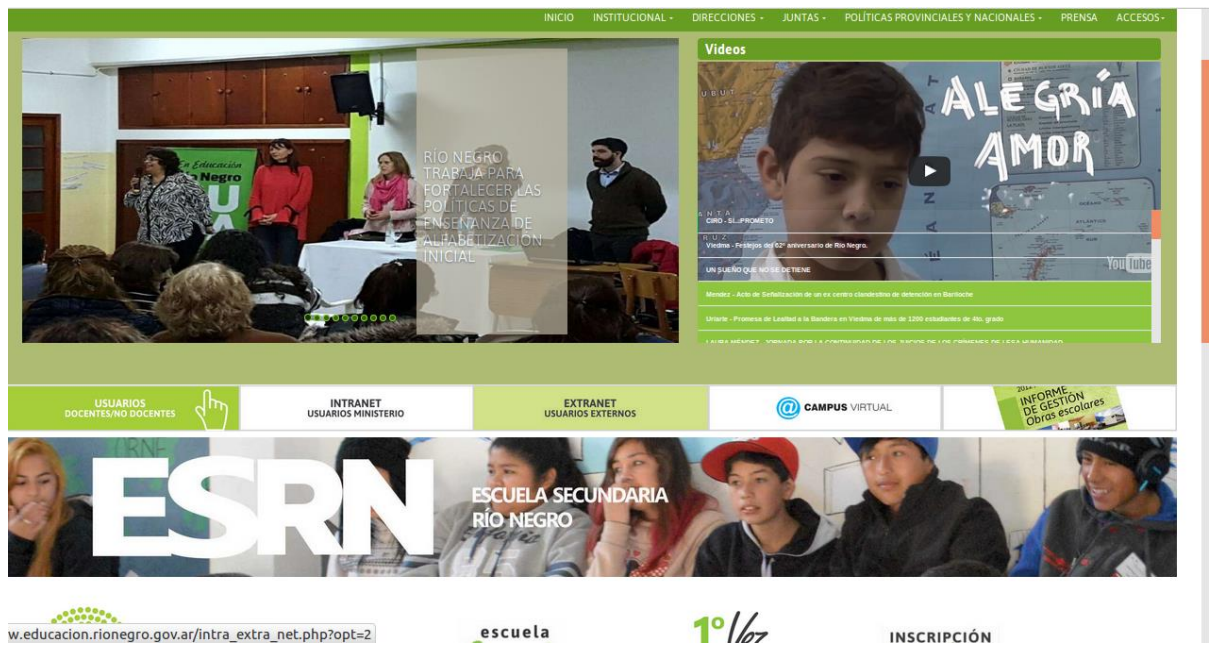

Figura 1. Captura de pantalla del sitio web de gobierno analizado

La provincia de Río Negro dispone de una superficie territorial muy amplia (203.013 km2), pero con una densidad poblacional de 3.10 habitantes por kilómetro cuadrado. Geográficamente se puede dividir en tres regiones: atlántica, valles (alto y medio) y andina totalizando 39 municipios. Siendo las ciudades más pobladas San Carlos de Bariloche (andina), General Roca (alto valle) y Viedma (atlántica). Esta última es la capital de la provincia y donde se centraliza la actividad de gobierno. Las distancias que separan a las diferentes ciudades son extensas, ejemplo entre San Carlos de Bariloche y la capital provincial hay más de $800 \mathrm{~km}$. Cuenta con 931 establecimientos educativos (82\% estatales y el resto privados), con una matrícula total de 216.103 estudiantes (Estadísticas Educativas Anuario 2015), distribuidos por niveles como se muestra en la figura 2.

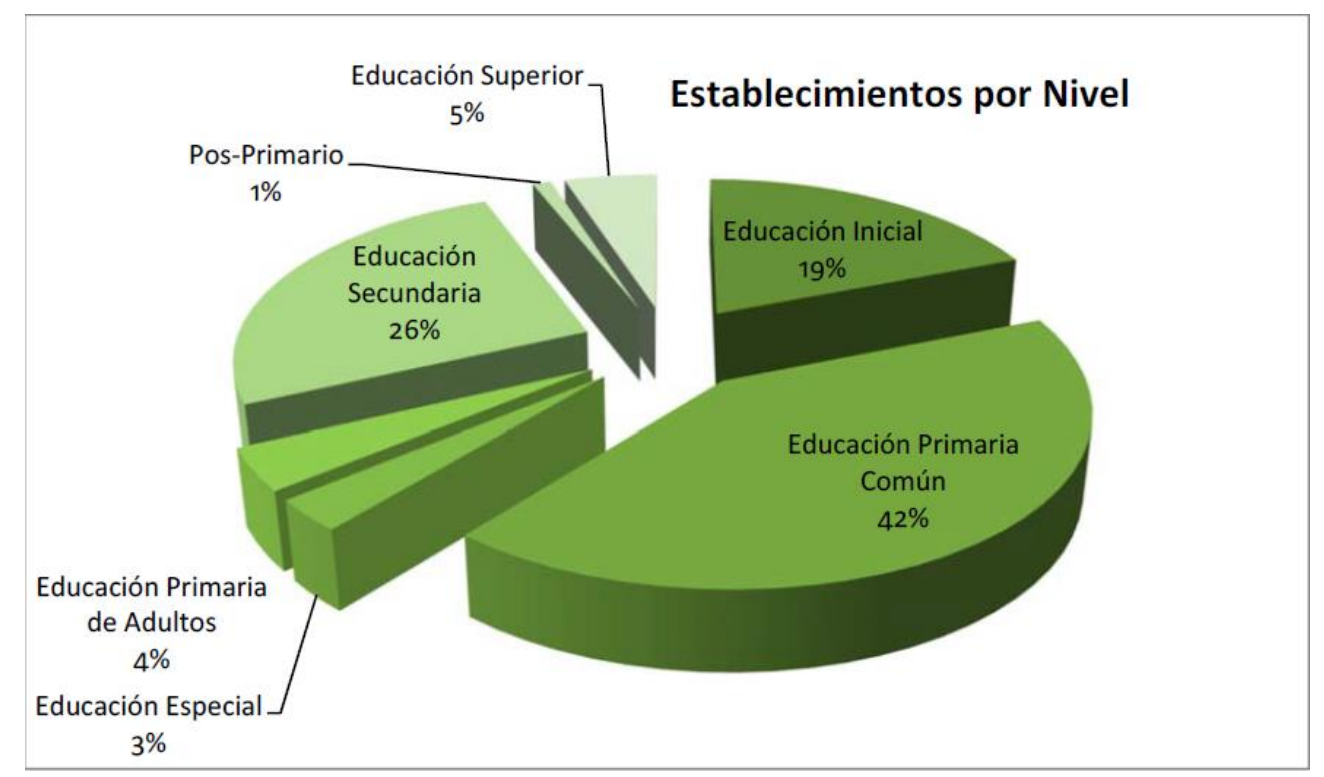

Figura 2. Distribución de establecimientos escolares por nivel (Fuente: Estadísticas Educativas Anuario, 2015) 
Para llevar adelante el análisis del discurso del sitio web de gobierno, se realizó una recolección de información a través de entrevistas virtuales y presenciales, a un grupo de miembros de la comunidad educativa, es decir usuarios destinatarios del sitio web. La Ley Orgánica de Educación de la provincia de Río Negro (2012), define como comunidad educativa a todos los sujetos intervinientes en el acto educativo: estudiantes, docentes, personal no docente, padres, madres, familias y adultos responsables

La recolección y análisis, se hizo de acuerdo a lo establecido en el Manual Operativo de Magariños de Morentín (1996). Se parte de realizar una pregunta particular a una muestra seleccionada y a partir del discurso obtenido, se analiza lo efectivamente dicho en el discurso social. Esto no depende inicialmente de las concepciones del entrevistador acerca de un fenómeno social sino de la preexistencia de un formación discursiva, que de cierta forma determina el decir individual, en tanto se convierte en la condición de posibilidad para cada miembro de una determinada comunidad a la cual pertenece cada productor del texto semiótico en cuestión (Magariños, 1996).

La pregunta que se formuló para este trabajo fue: ¿qué experiencia tiene con el sitio web del Ministerio de Educación de Río Negro? , de manera que fuera global. La muestra se conformó con un total de 20 entrevistas distribuidas entre la comunidad educativa de la ciudades de General Roca, Viedma y Bariloche. La selección de las localidades, siguió como criterio atender ciudades populosas de la provincia con diferentes realidades, desde su aspecto económico, cultural y por ende su comunidad educativa.

Previo al análisis, se realizó una búsqueda de aplicativos de software que permitieran sistematizar la información proveniente de las respuestas de los usuarios y de las usuarias, y realizar su análisis. Los aplicativos analizados son: T-LAb, QDA Miner, NVivo. Entre las limitaciones que presentan los mismos, se encuentran por una parte, el costo económico que exigen las licencias de uso, restricciones sobre las plataformas de uso, sumado al tiempo que demanda aprender a utilizarlos, más aún cuando los manuales de ayuda en algunos casos no están disponibles en español. En este sentido se optó por llevar adelante el análisis en forma manual de la información teniendo en cuenta que el tamaño de la muestra así lo permitía.

\section{Resultados}

A partir del análisis preliminar de las entrevistas realizadas, se han determinado cuatro ejes principales que conforman la formación discursiva relativa al sitio web: Pagina, Educación, Tipos de Interface y Comunidad Educativa. Estos a su vez se descomponen en otros sub-ejes que detallaran concretamente las diferentes semantizaciones que surgen del análisis del discurso de los distintos actores recortados. Esto puede visualizarse a través del árbol invertido 
de las representaciones/interpretaciones de la formación discursiva, que se presenta en la figura 3.

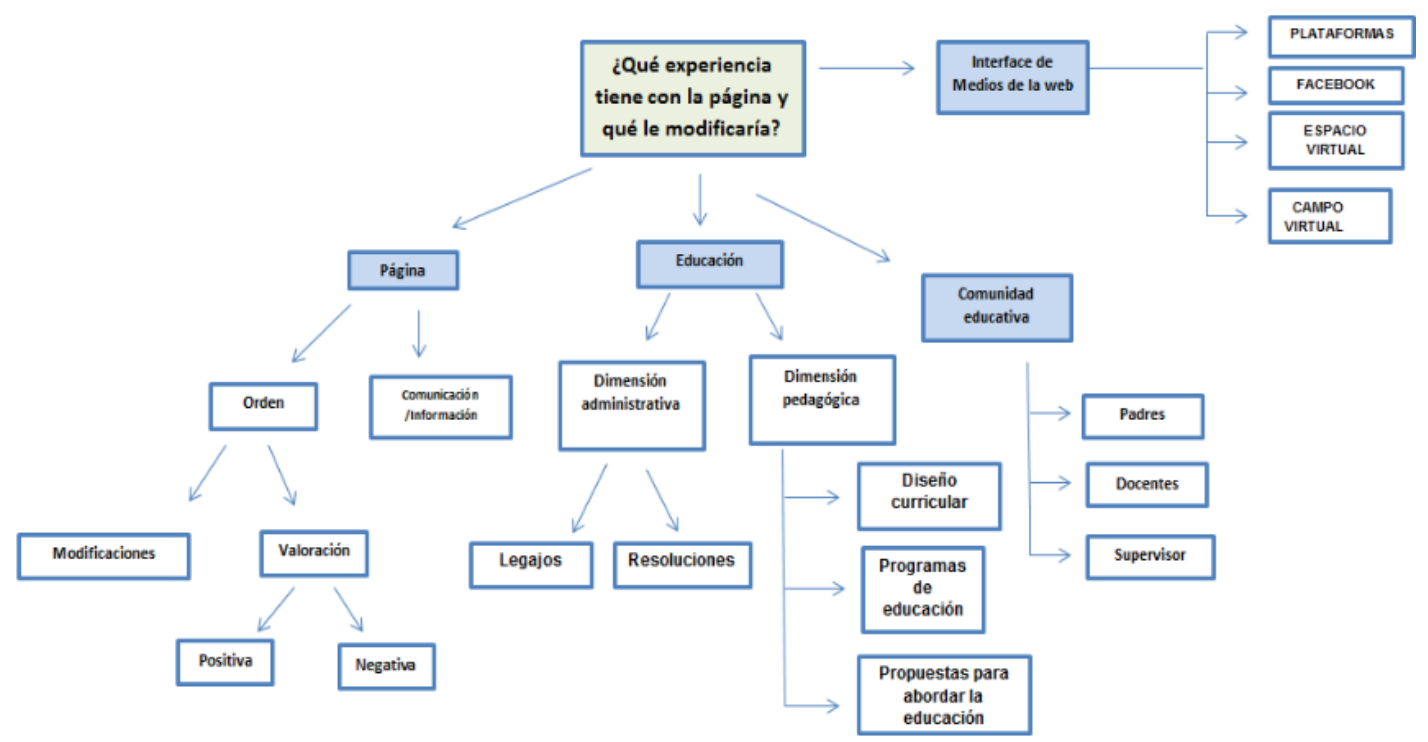

Figura 3. Árbol invertido de representaciones / interpretaciones de la formación discursiva.

A continuación se presenta un análisis de cada uno de los ejes principales a partir de lo efectivamente dicho en el discurso analizado (entrevistas):

-Página: el sitio presenta cierto desorden en cuanto a la información que describe, los menús desplegables son extensos (más de 9 opciones) lo cual dificulta el acceso a la información. La página de inicio, tiene una sobrecarga de información y en cuanto a las noticias la mayoría tratan sobre infraestructura o propagandas políticas del partido al que pertenece el gobierno actual. Así mismo, el análisis de la entrevistas, resalta el hecho de la falta de un buscador dentro del sitio web, que permita el acceso rápido a cierta información, lo cual agilizaría la búsqueda teniendo en cuenta la cantidad de información que presenta el sitio.

-Educación: este eje queda definido por dos dimensiones, ambas vinculadas al quehacer administrativo: una referida a la planificación sobre lo pedagógico y otra estrictamente vinculada al manejo de legajos o resoluciones. Esto hace suponer que el contenido está diseñado para proveer a los docentes de información administrativa necesaria en la cotidianidad de las escuelas. En este sentido, algunos de los entrevistados y las entrevistadas manifestaron la necesidad de diversificar la información de manera que sea útil a todos los destinatarios (Ej. cómo obtener un certificado de escolaridad, herramientas de acompañamiento escolar para el hogar, etcétera). 
-Comunidad educativa: al igual que en el anterior, se observa una demanda por parte de un grupo de usuarios (padres, personal no docente) respecto a la información que se presenta en el mismo.

-Tipos de Interface de medios: aquí se mencionan como medios de comunicación entre el Ministerio de Educación y Derechos Humanos de la provincia de Río Negro y los usuarios: a Facebook, y las plataformas de educación a distancia. Sobre este punto los usuarios manifiestan una falta de correlación de información en muchas ocasiones entre los diferentes medios. Así mismo, consideran que la red social Facebook no es un espacio formal para comunicar ciertas noticias o información importante vinculada a la tarea educativa. Por otra parte, en las entrevistas los usuarios manifiestan la necesidad de un espacio de comunicación interactivo entre los usuarios y el Ministerio.

\section{Discusión de Resultados}

En relación al uso del sitio puede observarse cierta disconformidad de los usuarios y de las usuarias respecto a la forma de presentar la información (menús y submenús) y a la falta de recursos para buscar información cuando no se sabe que camino tomar para ubicarla. Situaciones como estas dificultan la usabilidad del sitio y podrían estar asociadas como señalan Díaz et al. (2013) a la falta de normas para llevar adelante el diseño del sitio. Por otra parte, algunos usuarios (como padres, alumnos) tienen necesidades de información no cubiertas por el sitio, esto hace pensar en la necesidad de revisar perfiles de usuarios y diseñar o revisar el diseño del sitio en función de la información y expectativas de los diferentes perfiles de usuarios (Proyecto ProGobernabilidad, 2013).

Respecto a la comunicación, el análisis muestra que los usuarios demandan un sitio web más interactivo, que posibilite una nueva relación entre el Ministerio y la comunidad educativa en su conjunto. En este sentido, se podrían mencionar las reflexiones realizadas por Ceraso (2006) y Uranga (2008), que nos permiten pensar cómo ha variado la comunicación en estos tiempos y la importancia de dar al usuario un lugar para poder ser partícipe de la misma, dejando de lado formas de comunicación vertical como la que perciben los usuarios del sitio web analizado.

En cuanto al acceso a otros canales de comunicación desde el sitio (Facebook por ejemplo) resultan interesantes como nuevas posibilidades para que el usuario interactúe con el organismo de gobierno (Rodríguez et al., 2015), en la medida que la información sea consistente en todos los medios y la comunicación no sea solo en un sentido.

\section{Conclusiones}


Desde la teoría de la aguja hipodérmica hasta nuestros días, la comunicación se ha transformado, incrementando la capacidad de poder generar un intercambio entre las partes, con la intención de producir sentido, teniendo en cuenta que la verdad no es única ni absoluta a la hora de comunicar, sino que es un acto conjunto que hace que los diferentes actores se entrelacen o entrecrucen miradas que sean formadoras de opinión e intercambio (Ceraso, 2006). Siguiendo este posicionamiento y en base a los resultados alcanzados hasta aquí con el análisis del discurso del grupo de usuarios entrevistados (miembros de la comunidad educativa), consideramos que resulta relevante pensar un re-diseño del sitio web analizado, de manera que este se convierta en un espacio para la interacción dando lugar al intercambio de opiniones sin que ello implique discusiones desvirtuadas sino por el contrario, que sean productoras de sentido y posibiliten que los ciudadanos, en nuestro caso, comunidad educativa, cuenten con recursos que les permitan incidir en la gestión de lo público en tiempos de gobierno electrónico (Líppez-De Castro y García, 2016).

Es importante señalar el carácter preliminar de los resultados presentados y discutidos en este artículo. En este sentido, se propone a futuro realizar un segundo análisis que incluya otras realidades educativas de la provincia de Río Negro, como son los Centros de Educación Media Rural (CEM), así como también ampliar el tamaño de la muestra. Sobre este punto, se profundizará en la búsqueda de un aplicativo de software que permita sistematizar la información recolectada, en forma libre y gratuita.

\section{Bibliografía}

Amadeo, B. (2007). Comunicación Gubernamental e Internet en la República Argentina. Comunicación en la Academia Nacional de Ciencias de Buenos Aires en la sesión privada extraordinaria del 18 de octubre de 2007.

Castro, M.; Dasso, A. y Funes, A. (2009). Evaluación de atributos de sitios de gobierno electrónico. XI Workshop de Investigadores en Ciencias de la Computación, Universidad Nacional de San Juan.

Ceraso, C. (2006). Sembrando mi tierra de futuro. La Plata, Bs. As.: Ediciones de Periodismo y Comunicación, Facultad de Periodismo y Comunicación Social (UNLP).

Díaz, F. J.; Harari, I. y Amadeo, A. P. (2013). Guía de recomendaciones para diseño de software centrado en el usuario. La Plata: Editorial de la Universidad Nacional de La Plata (EDULP). 
Ministerio de Educación y Derechos Humanos de la Provincia de Rìo Negro (2015). Estadísticas Educativas Anuario. Recuperado de www.campus1.rionegro.gov.ar/descargar.php?id=7284

Giulianelli, D. A.; Rodríguez, R. A.; Vera, P. M. y Welicki, L. E. (2008). Situación Global de Gobernabilidad Electrónica en Sitios Web Municipales. Conferencia CollECTeR Iberoamérica, Madrid. Recuperado de http://www. researchgate. net/publication/228401237_Situacin_Global_de_Gobernabilid ad_Electrnica_en_Sitios_Web_Municipales.

Giulianelli, D. A.; Rodríguez, R. A.; Vera, P. M.; Trigueros, A. y Marko, I. (2013). Los sitios web gubernamentales como facilitadores del gobierno electrónico. XV Workshop de Investigadores en Ciencias de la Computación, Universidad Nacional de San Juan.

Harris, Z. S. (1954). Distributional structure. Word, 10(2-3), pp. 146-162.

Ley Orgánica de Educación de la Provincia de Río Negro. (2012). Recuperado de www3.educacion.rionegro.gov.ar/.../Nueva_Ley_de_Organica_de_Educacion.pdf

Líppez-De Castro, S. y García, R. (2016). Ciudadanos y gobierno electrónico: la orientación al ciudadano de los sitios Web municipales en Colombia para la promoción de la participación. Universitas Humanística, 279-304. http://dx.doi.org/10.11144/Javeriana.uh82.cgeo

McLuhan, M. (1964). Understanding media: The extensions of man. Nueva York: New American Library.

Magariños de Morentin, J. (1996). Manual Operativo para la aplicación de la Semiótica de Enunciados. OPS, Programa Mujer, Salud y Desarrollo.

Pecheux, M. (1969). Analyse automatique du discours. Paris: Dunod.

Pecheux, M. (1975). Les vérités de La Palice. Paris: Maspéro.

Reynoso, L. A.; Vaucheret, C. A.; Grosso, G.; Amaolo, M. P.; Szneck, J.; Dolz, D. y Kazalukian, M. (Junio de 2013). Modelos y tecnologías en gobierno electrónico. XV Workshop de Investigadores en Ciencias de la Computación, Universidad Nacional de San Juan.

Rodríguez, R. A. (2011). Marco de Medición de calidad para gobierno electrónico (Doctoral dissertation), La Plata: Facultad de Informática, Universidad Nacional de La Plata.

Rodríguez, R.; Vera, P. y Marko, I. (2015). El Gobierno Electrónico y la Implementación de las TIC para Brindar Nuevos Canales de Comunicación. Revista Latinoamericana de Ingeniería de Software, 3(5), pp. 187-196.

Scolari, C. A. (Ed.). (2015). Ecología de los medios: entornos, evoluciones e interpretaciones. España: Editorial GEDISA.

Uranga, W. (2009). El sentido de las técnicas en el diagnóstico desde la comunicación. Algunos ejemplos y propuestas para trabajar, material de la cátedra de Planificación de 
Procesos comunicacionales. La Plata: Facultad de Periodismo y Comunicación Social, Universidad Nacional de La Plata. La Plata.

Vílchez, L. (2001). La migración digital. Barcelona: Gedisa. 